\title{
Disseminated cancer cells in the bone marrow: are they really there at all?
}

\author{
YASUHIRO KODERA \\ Department of Surgery II, Nagoya University Graduate School of Medicine, 65 Tsurumai-cho, Showa-ku, Nagoya 466-8550, Japan
}

Since the early 1990s, several investigators have turned to molecular diagnosis by immunostaining and reversetranscriptase polymerase chain reaction (RT-PCR) to detect minimal residual disease and occult metastasis in various body components, including lymph nodes [1,2], peritoneal washes $[1,3]$, peripheral blood, and bone marrow aspirates $[1,4,5]$. In the case of gastric cancer, opinions regarding the prognostic significance of cancer cells in each of these components have been mixed, and only detection in the peritoneal washes has been consistently reported as being of value. Peritoneal carcinomatosis is a frequently observed consequence of advanced gastric cancer, and free cancer cells in the peritoneal washes detected by conventional Papanicolaou staining denote poor prognosis even in the absence of visible peritoneal deposits [6]. Here, molecular diagnosis succeeded in improving the sensitivity of the cytologic examination, possibly because (1) even the isolated tumor cells (ITCs: metastases and cancer cell aggregates measuring less than $0.2 \mathrm{~mm}$ in size) could survive and metastasize in this particular environment and (2) the quantity and variety of non-cancer cells that contaminate the samples are limited in comparison with components such as the peripheral blood and bone marrow aspirates, rendering these samples relatively easy targets for RT-PCR.

The prognostic value of detecting minimal disease is somewhat more controversial in the case of nodal metastasis. Here, so-called ITCs and micrometastases (metastases measuring less than $2 \mathrm{~mm}$ in size) seem to behave differently. It has been reported that in an in vivo model of lymph node metastasis using a gastric cancer cell line, nodal ITCs regress spontaneously following resection of the primary, whereas nodal micrometastases grow to gross metastases after surgery [7]. This phenomenon seems to justify the International Union Against Cancer (UICC) classification that

Offprint requests to: Y. Kodera categorizes ITCs as pN0 and micrometastases as pN1mi. However, discrimination between ITCs and micrometastases is by no means straightforward even when attempted by experienced pathologists [8]. In addition, a search for minimal nodal disease is usually performed with lymph nodes that have been resected. In other words, the investigators are not looking at what has been left behind as residual disease, but are simply scrutinizing the nodal status using surgically resected specimens.

When it comes to bone marrow aspirates, the prognostic significance of detecting cancer cells seems to be even more ambiguous. Ten years ago, surgeons were alarmed by a report from Maehara et al. that cancer cells were found by immunostaining in the bone marrow of two patients with mucosal gastric cancer [4]. They proceeded to find cancer cells in the bone marrow of 9 of 45 patients $(20 \%)$ with early gastric cancer. Given the excellent prognosis of early gastric cancer, however, one has to reach either of the two following conclusions: (1) unlike for other cancers, cancer cells in the bone marrow have little clinical implication for gastric carcinoma; or (2) what they found in the bone marrow were not cancer cells after all. In theory, bone marrow could harbor dormant cancer cells that could result in cancer relapse after several years of latency. This may perhaps apply to breast cancer, in which late recurrences are not uncommon. However, tumor dormancy is seldom reflected in the natural history of gastric cancer, in which $70 \%$ of the recurrent disease is detected within 2 years of curative surgery [9].

In this issue of Gastric Cancer, Fujita et al. report on yet another attempt to detect cancer cells in the bone marrow aspirates of gastric cancer patients [10]. Although I do not wish to be considered an old man who refuses to believe what he cannot see with his own eyes, RT-PCR continues to have an intrinsic weakness in that it is not necessarily looking at cancer cells-that the mRNA it detects is not always specific to neoplastic 
cells. Clinical samples are invariably contaminated with various non-neoplastic cells, and the accuracy of RTPCR would presumably correlate inversely with the amount and variety of non-cancer cells contained in the sample. From this point of view, detection of cancer cells from the bone marrow aspirates, packed with numerous blood cell components and progenitors, could be a formidable challenge. Sensitivity of RT-PCR could be improved by increasing the cycle number employed in the protocol, but the higher the cycle number is raised, the higher the probability of detecting minute mRNAs contained in non-cancer cells would be, resulting in high false-positive results. At least two authors have maintained that lowering the cycle number adequately has deprived RT-PCR of a sufficient sensitivity for it to replace immunostaining for evaluation of the peripheral blood [11], and the same could easily be the case with bone marrow aspirates. Other authors, in greater numbers, have maintained that sensitive detection of minimal disease from the peripheral blood and bone marrow is feasible and reflects clinical outcome [1], however, and this discrepancy remains an enigma. To say the least, establishment of the PCR protocol for evaluating the bone marrow aspirates and adjustment and maintenance of the detection system call for the utmost care. Alternation of personnel who handle the system or even of the chemicals used during the detection procedure could lead to catastrophic changes in the overall results. Possibly reflecting these facts, the detection rate of cancer cells in the bone marrow using CK20 as a target differed dramatically between Fujita et al. and another sample set reported from Maehara's group [5], despite little difference in patient demographics. Fujita et al. found only one of 65 patients to be positive in their examination, whereas Oki et al. stated that the mRNA level exceeded their cutoff value in 27 of 47 patients [5]. The results obtained by Oki et al. conform to those of several of the previous studies in which gastric cancer cells were found not to be entirely foreign in the bone marrow [1]. Their presentation is not accompanied by corresponding survival data, however, and justification of the high detection rate in association with the clinical outcome is yet to be confirmed. In contrast, the results of Fujita et al. are more believable, given the clinical outcome of gastric cancer that we are generally acquainted with. However, their findings render PCR detection of cancer cells in the bone marrow meaningless, despite numerous attempts and successes reported from other groups. This is an awkward situation for an editor who has to take into account the social impact of an article along with its scientific quality.

Studying the incidence and consequences of various micrometastases may help one to gain insight into the biology of cancer. However, we will have to admit through reading over these conflicting data that this is not the right time to use the information on cancer cells in the bone marrow for any decision making regarding the treatment of gastric cancer. Evidence in support of routine application of adjuvant treatments for curatively resected advanced cancer has finally begun to emerge from Asia, and we are currently expecting more big news to come. It is time to move on with a new standard of care and continue to seek better results, first by further improving on the treatment to be given and contemplating when it should be started and how long it should last. We hope that a more refined technique will be established in the meantime to adequately identify patients with relevant residual disease.

\section{References}

1. Wolfrum F, Vogel I, Fandrich F, Kalthoff H. Detection and clinical implications of minimal residual disease in gastro-intestinal cancer. Langenbecks Arch Surg 2005;390:430-41.

2. Fukagawa T, Sasako M, Mann GB, Sano T, Katai H, Maruyama $\mathrm{K}$, et al. Immunohistochemistry detected micrometastases of the lymph nodes in patients with gastric carcinoma. Cancer 2001;92: 753-60.

3. Kodera $\mathrm{Y}$, Nakanishi $\mathrm{H}$, Ito $\mathrm{S}$, Mochizuki $\mathrm{Y}$, Ohashi $\mathrm{N}$, Yamamura $\mathrm{Y}$, et al. Prognostic significance of intraperitoneal cancer cells in gastric carcinoma: analysis of real-time reverse transcriptase-polymerase chain reaction after 5 years of followup. J Am Coll Surg 2006;202:231-6.

4. Maehara Y, Yamamoto M, Oda S, Baba H, Kusumoto T, Ohno S, et al. Cytokeratin-positive cells in bone marrow for identifying distant micrometastasis of gastric cancer. Br J Cancer 1996;73:837.

5. Oki E, Maehara Y, Tokunaga E, Shibahara K, Hasuda S, Kakeji $\mathrm{Y}$, et al. Detection of disseminated cancer cells in bone marrow of gastric cancer using real-time quantitative reverse transcriptase polymerase chain reaction. Cancer Lett 2002;188:191-8.

6. Kodera Y, Yamamura Y, Shimizu Y, Torii A, Hirai T, Yasui K, et al. Peritoneal washing cytology: prognostic value of positive findings in patients with gastric carcinoma undergoing a potentially curative resection. J Surg Oncol 1999;72:60-4.

7. Yokoyama H, Nakanishi H, Kodera Y, Ikehara Y, Ohashi N, Ito $\mathrm{Y}$, et al. Biological significance of isolated tumor cells and micrometastasis in lymph nodes evaluated using a green fluorescent protein-tagged human gastric cancer cell line. Clin Cancer Res 2006;12:361-8.

8. Cserni G, Bianchi S, Boecker W, Decker T, Lacerda M, Rank F, et al. Improving the reproducibility of diagnosing micrometastases and isolated tumor cells. Cancer 2005;103:358-67.

9. Kodera Y, Ito S, Yamamura Y, Mochizuki Y, Fujiwara M, Hibi K, et al. Follow-up surveillance for recurrence after curative gastric cancer surgery lacks survival benefit. Ann Surg Oncol 2003;10: 898-902.

10. Fujita Y, Terashima M, Hoshino Y, Ohtani S, Kashimura S, Kanzaki N, et al. Detection of cancer cells disseminated in bone marrow using real-time quantitative RT-PCR of CEA, CK19 and CK20 mRNA in patients with gastric cancer. Gastric Cancer 2006;9:308-14.

11. Ko Y, Klinz M, Totzke G, Gouni-Berthold I, Sachinidis A, Vetter $\mathrm{H}$. Limitations of the reverse transcription-polymerase chain reaction method for the detection of carcinomembryonic antigenpositive tumor cells in peripheral blood. Clin Cancer Res 1998;4: 2141-6. 